\title{
Performance Characterization of High Gain, High Output Power And Low Noise Cascaded Broadband Discrete Raman Amplifiers
}

\author{
Md Asif Iqbal, Mingming Tan, Lukasz Krzczanowicz, Pavel Skvortcov, Atalla El-Taher, Ian D. Philips, \\ Wladek Forysiak, Juan Diego Ania-Castañón* and Paul Harper \\ AIPT, Aston University, Birmingham B4 7ET, UK \\ * Instituto de Óptica "Daza de Valdés”, CSIC, 28006 Madrid, Spain \\ Tel: (44) 121204 3512,e-mail: iqbalm7@aston.ac.uk
}

\begin{abstract}
In this paper, gain, noise and nonlinear performance characterization of cascaded broadband discrete Raman amplifiers with different gain fibre combinations is presented. We numerically demonstrate the design of a backward-pumped cascaded dual stage broadband $(\sim 70 \mathrm{~nm})$ discrete Raman amplifier with high gain $(\sim 19 \mathrm{~dB})$, low noise ( $\sigma 6 \mathrm{~dB}$ noise figure) and lower nonlinear penalty by optimizing two different types of gain fibres.
\end{abstract}

Keywords: Discrete Raman amplifier, noise figure, nonlinear phase shift.

\section{INTRODUCTION}

Discrete Raman amplifiers can seamlessly extend the transmission bandwidth into any spectral region of fibre by using suitable pump wavelengths [1,2], compared to conventional erbium-doped fibre amplifier (EDFA) which is limited to mainly $\mathrm{C}$ or $\mathrm{L}$ band. To improve the pump conversion efficiency, gain fibres with high Raman gain co-efficient and small core area are usually preferred in discrete Raman amplifiers. Dispersion compensating fibre (DCF), highly nonlinear fibre (HNLF) and dispersion shifted fibre (DSF) have already been used due to their high Raman gain properties [1-3]. Inverse dispersion fibre (IDF) has recently been proven as an alternative of DCF for in-line dispersion and nonlinearity management [4,5].

The challenges of using these gain fibres are double Rayleigh backscattering (DBS) induced multipath interference (MPI) and nonlinear impairments due to high nonlinear index of fibre. DBS significantly deteriorates the optical signal to noise ratio (OSNR) performance and sets an upper limit to the maximum gain of the amplifier. Separating the gain fibre lengths into two stages through an isolator [6], or amplifying in dual stage configurations with the same set of pump wavelengths have already been presented [7]. The main drawbacks of those configurations were poor pump conversion efficiency, as very high pump powers were needed to amplify both stages by reusing residual pumps, and large gain variations due to less flexibility of gain spectra control at each stage. Moreover, nonlinear penalty, which could be detrimental due to the use of significantly longer lengths of the same gain fibre with very high nonlinearity in both stages, has not been analysed. Nonlinear penalty also increases with the length and the nonlinear refractive index parameter of the gain fibre, so proper choice of gain fibre length and nonlinear efficiency are required to optimize DBS and nonlinear penalty in designing discrete Raman amplifiers with high gain, low noise and nonlinear penalty.

In this paper, we numerically demonstrate a design of broadband discrete Raman amplifiers in dual stage cascaded configuration pumping with only four backward $1^{\text {st }}$ order pumps. We report $19 \mathrm{~dB}$ average net gain over $70 \mathrm{~nm}$ bandwidth, $>20 \mathrm{dBm}$ output power, $\sim 6 \mathrm{~dB}$ noise figure and low nonlinear penalty. We investigated the performances of three different, commonly used Raman gain fibres: DCF, HNLF and IDF, in different combinations, and found that cascading HNLF $2 \mathrm{~km}$ and IDF $6 \mathrm{~km}$ as $1^{\text {st }}-2^{\text {nd }}$ stage configuration gave the best noise and nonlinear performance. Nonlinear impairment was calculated by determining the self-phase modulation (SPM) induced nonlinear phase shift (NPS) [3]. Noise performance was characterized by noise figure (NF) and output OSNR considering combined amplified spontaneous emission (ASE) and DRB noise contribution.

\section{PROPOSED AMPLIFIER DESIGN AND SIMULATION SETUP}

The amplifier configuration is shown in Fig. 1. It comprises two stages and different combinations of three different gain fibres DCF, HNLF and IDF were investigated in each stage. The fibre parameters are given in Table 1. We considered 70nm amplification bandwidth from 1530 to $1600 \mathrm{~nm}$. Only four depolarised cascaded $1^{\text {st }}$ order Raman pumps: $1427 \mathrm{~nm}, 1445 \mathrm{~nm}, 1462 \mathrm{~nm}$ and $1490 \mathrm{~nm}$ were considered in backward pumping configuration at each stage to achieve total gain ripple $\sim 1.6 \mathrm{~dB}$. Equal signal power per channel of $-10 \mathrm{dBm}$ was considered at the input of the amplifier and mid-stage insertion loss from WDM (wavelength division multiplexer) and isolator of $1 \mathrm{~dB}$ was considered for signals.

The complete numerical model for the evolution of signals, pumps and noise is based on standard average power model [8]. Pump depletion, ASE noise, DBS, energy transfer due to interactions between pump-signal and signal-signal from either direction have been included in the model. 


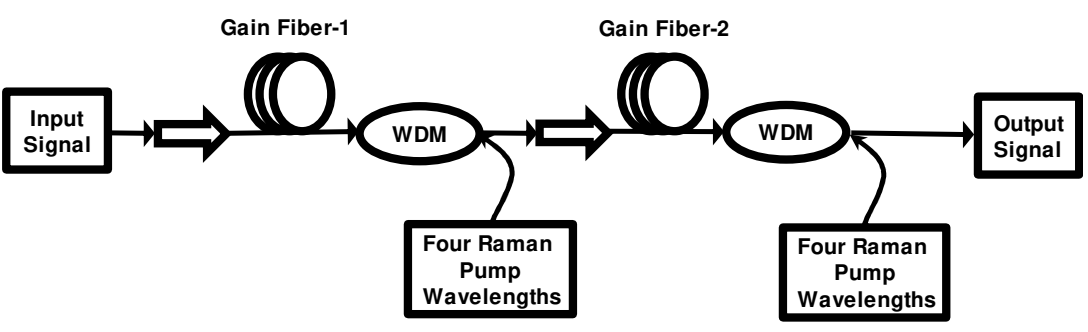

Fig. 1: Schematic diagram of cascaded dual stage discrete Raman amplifier.

\section{RESULTS AND DISCUSSION}

All the results were obtained through numerical simulation considering the amplifier configuration described in section 2. Firstly, the performance of discrete Raman amplifier was investigated in single stage configuration by varying the lengths of different gain fibres in order to compare their noise and nonlinear performances. The target average net gain was fixed at $\sim 10.8 \mathrm{~dB}$ in each case with $<1.5 \mathrm{~dB}$ gain ripple. Maximum pump power per wavelength was limited to $300 \mathrm{~mW}$. It was found that, noise figure (NF) performance degrades as the length of gain fibre increases in single stage configuration, due to increasing DBS induced MPI noise. On the other hand, total pump power requirement can be reduced with increasing amplifier length until attenuation loss becomes dominant over Raman gain efficiency.

Fig. 2 shows the characterization results with IDF fibre. The length of IDF is varied from $6 \mathrm{~km}$ to $16 \mathrm{~km}, 6 \mathrm{~km}$ being the lowest possible length with maximum $300 \mathrm{~mW}$ power at lowest wavelength $1427 \mathrm{~nm}$ pump. Both ASE and DRB contributions were taken into consideration in wavelength dependent NF and OSNR calculation. As expected, low wavelength signals have higher noise figure due to being close to the longer wavelength pumps and thus have more thermally generated noise. The tilt in noise figure could be minimised by forward pumping with shorter wavelength pumps in a bidirectional pumping configuration which is out of the scope of this paper. In Fig. 2(b) and (c), both NF and NPS increase with increasing lengths of IDF gain fibre, thanks to the impact of DBS and total average power along the span respectively. Output OSNR follows the opposite trend of NF as shown in Fig. 2(d). So, discrete Raman amplifier with shorter gain fibre length is the best in terms of noise and nonlinear performance but at the expense of pump power efficiency.

Similar characterisations were carried out for HNLF and DCF at different lengths: $2 \sim 5.5 \mathrm{~km}$ and $5 \sim 12.5 \mathrm{~km}$ respectively with the same target net gain, gain ripple and pump power constraints. Similar trends in NF, NPS and output OSNR have also been observed, as shorter lengths being the best choice for low noise and NPS induced penalty. Pump powers used in the best possible lengths of each gain fibre are given in Table 2.
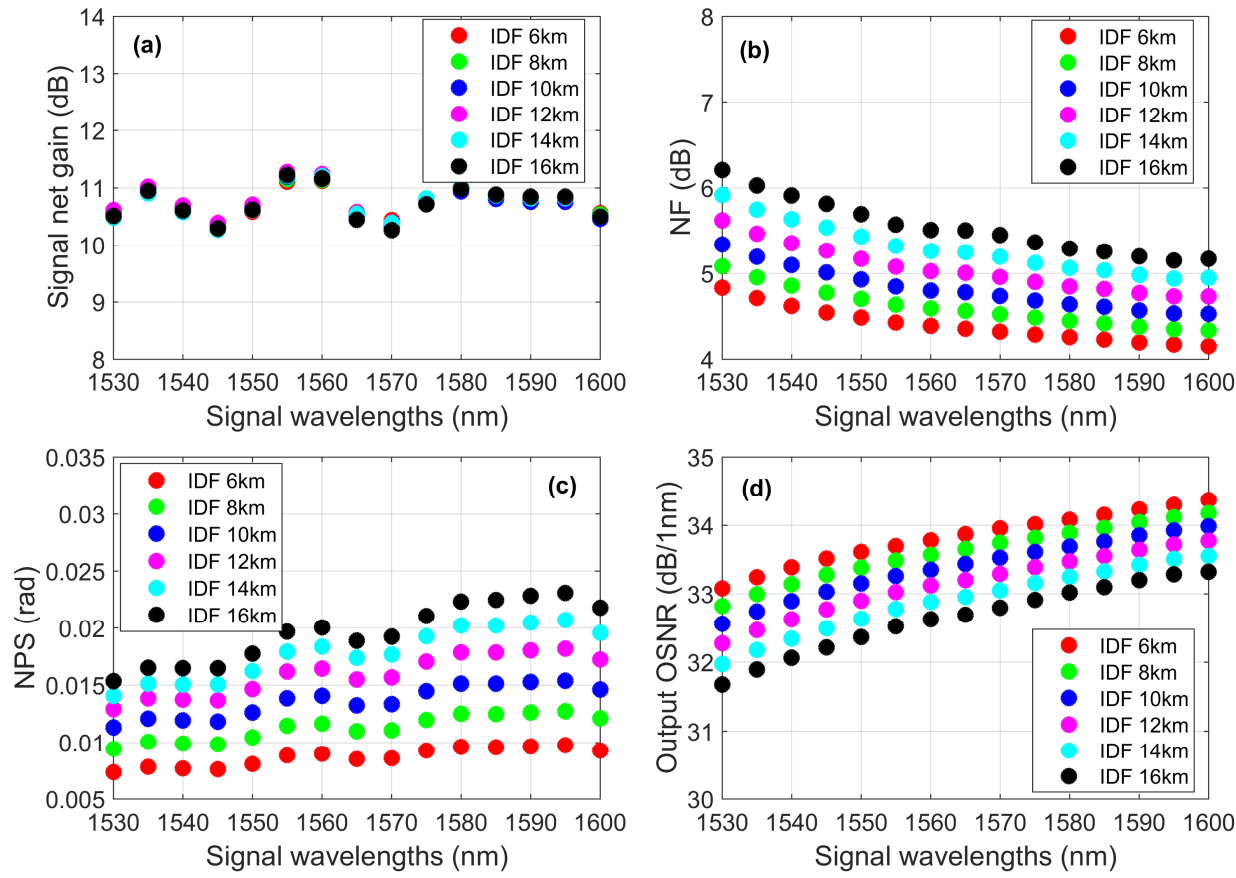

Fig. 2: Characterizations of IDF based single stage discrete Raman amplifier with varying lengths (a) signal net gain (b) noise figure (c) nonlinear phase shift and (d) output OSNR 
Table 1. Summary of fibre parameters.

\begin{tabular}{|c|c|c|c|}
\hline Parameters & HNLF & IDF & DCF \\
\hline$\alpha_{1455}(\mathrm{~dB} / \mathrm{km})$ & 0.75 & 0.3 & 0.8 \\
\hline$\alpha_{1550}(\mathrm{~dB} / \mathrm{km})$ & 0.6 & 0.23 & 0.67 \\
\hline $\mathrm{A}_{\mathrm{eff}}\left(\mu \mathrm{m}^{2}\right)$ & 10.2 & 31 & 19 \\
\hline $\mathrm{g}_{\mathrm{R}} / \mathrm{A}_{\mathrm{eff}}\left(\mathrm{W}^{-1} \mathrm{~km}^{-1}\right)$ & 6.3 & 1.3 & 2.5 \\
\hline$\kappa\left(10^{-4} \mathrm{~km}^{-1}\right) @ 1550 \mathrm{~nm}$ & 9.2 & 1.2 & 7.1 \\
\hline $\mathrm{n}_{2}\left(10^{-20} \mathrm{~m}^{2} / \mathrm{W}\right)$ & 4.49 & 2.7 & 3.95 \\
\hline$\gamma\left(\mathrm{W}^{-1} \mathrm{~km}^{-1}\right)$ & 17.84 & 3.53 & 8.43 \\
\hline $\begin{array}{c}\text { Dispersion }(\mathrm{ps} / \mathrm{nm} / \mathrm{km}) \\
@ 1550 \mathrm{~nm}\end{array}$ & -20.7 & -44 & -100 \\
\hline
\end{tabular}

Table 2. Pump powers used in single stage.

\begin{tabular}{|c|c|c|c|}
\hline \multirow{2}{*}{$\begin{array}{c}\text { Pumps } \\
(\mathrm{nm})\end{array}$} & \multicolumn{3}{|c|}{ Pump power $(\mathrm{mW})$} \\
\cline { 2 - 4 } & $\begin{array}{c}\text { HNLF } \\
2 \mathrm{~km}\end{array}$ & $\begin{array}{c}\text { IDF } \\
6 \mathrm{~km}\end{array}$ & $\begin{array}{c}\text { DCF } \\
5 \mathrm{~km}\end{array}$ \\
\hline 1427 & 205 & 300 & 290 \\
\hline 1445 & 95 & 185 & 180 \\
\hline 1462 & 90 & 100 & 110 \\
\hline 1490 & 113 & 200 & 165 \\
\hline Total & 503 & 785 & 745 \\
\hline
\end{tabular}

Performance comparison of three different gain fibres in single stage configuration with the best performing lengths is shown in Fig. 3. NF and output OSNR over 70nm bandwidth are almost similar for $6 \mathrm{~km}$ IDF and $2 \mathrm{~km}$ HNLF whereas $5 \mathrm{~km}$ DCF shows more than $1 \mathrm{~dB}$ penalty as shown in Fig. 3(a) and (b). Although HNLF shows similar noise performance as IDF but has worse nonlinear penalty that is similar to DCF as depicted in Fig. 3(c). Here, although DCF has lower nonlinear refractive index than HNLF as mentioned in Table 1 but due to higher average power over longer length makes the nonlinear penalty as much as HNLF with half of the length. However, IDF has the lowest nonlinear penalty due to its larger effective area and lower nonlinear co-efficient than DCF and HNLF.
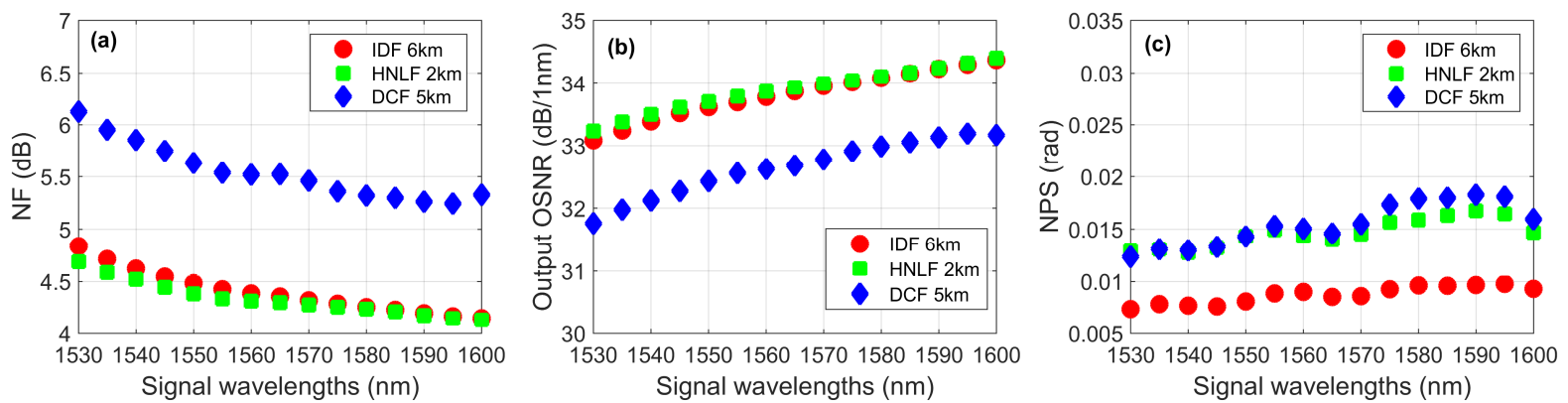

Fig. 3: Performance comparison of different single stage discrete Raman amplifiers with average target net gain of 10.8dB and at optimum length considered (a) noise figure (b) output OSNR and (c) nonlinear phase shift.

Total noise performance in a cascaded amplifier chain mainly depends on the noise contribution from the $1^{\text {st }}$ stage amplifier. Usually higher net gain and lower noise figure are considered in the $1^{\text {st }}$ stage to get better overall performance. Here, only IDF $6 \mathrm{~km}$ and HNLF $2 \mathrm{~km}$ have been used as the $1^{\text {st }}$ stage which allowed us to investigate six different possible combinations of dual stage discrete Raman amplifier. The target average net gain from the combined dual stages was set to $19 \mathrm{~dB}$ with a maximum ripple of $1.6 \mathrm{~dB}$.

Fig. 4(a) shows the net gain and output power of signals in all the dual stage configurations considered here. It can be seen that, average total net gain is almost the same $(\sim 19 \mathrm{~dB})$ and average total output signal power is $>20 \mathrm{dBm}(\sim 9 \mathrm{dBm} / \mathrm{channel})$ for all the schemes. Fig. 4(b) shows that all dual stage configurations with HNLF $2 \mathrm{~km}$ based $1^{\text {st }}$ stage (HNLF $2 \mathrm{~km}$ - IDF $6 \mathrm{~km}$, HNLF $2 \mathrm{~km}$ - HNLF $2 \mathrm{~km}$ and HNLF $2 \mathrm{~km}$ - DCF $5 \mathrm{~km}$ ) have better noise figure than its counterpart IDF $6 \mathrm{~km} 1^{\text {st }}$ stage based configurations because of having slightly better noise performance in the $1^{\text {st }}$ stage as shown in Fig. 3(a) and (b). As expected dual stage amplifiers with DCF 5km in $2^{\text {nd }}$ stage shows the worst noise performance regardless of their $1^{\text {st }}$ stage configuration. HNLF $2 \mathrm{~km}-$ IDF $6 \mathrm{~km}$ shows the minimum noise figure $(\sim 6 \mathrm{~dB})$ with less than $1 \mathrm{~dB}$ tilt across the $70 \mathrm{~nm}$ bandwidth.

Total nonlinear phase shift for all signal wavelengths have also been calculated as shown in Fig. 4(c). Nonlinear phase shift depends both on the nonlinear co-efficient of gain fibre and average signal power along the length of amplifier. Although IDF $6 \mathrm{~km}$ shows better nonlinear performance than HNLF $2 \mathrm{~km}$ in single stage characterization (Fig. 3(d)) but in all dual stage combinations (IDF 6km - IDF 6km, IDF 6km - HNLF $2 \mathrm{~km}$ and IDF $6 \mathrm{~km}$ - DCF $5 \mathrm{~km}$ ), it shows worse performance than HNLF $2 \mathrm{~km}$ counterpart. IDF $6 \mathrm{~km}-$ HNLF $2 \mathrm{~km}$ dual stage configuration has the highest nonlinear penalty, because HNLF has a nonlinear co-efficient $\sim 5$ times higher than IDF and average signal power is significantly higher in the $2^{\text {nd }}$ stage. Nonlinear penalty is the lowest with HNLF $2 \mathrm{~km}$ - IDF $6 \mathrm{~km}$ configuration because IDF has the lowest nonlinear co-efficient and the best single stage NPS performance than both DCF and HNLF. Fig. 4(d) shows the signal power evolution of all dual stage amplifier configurations. 

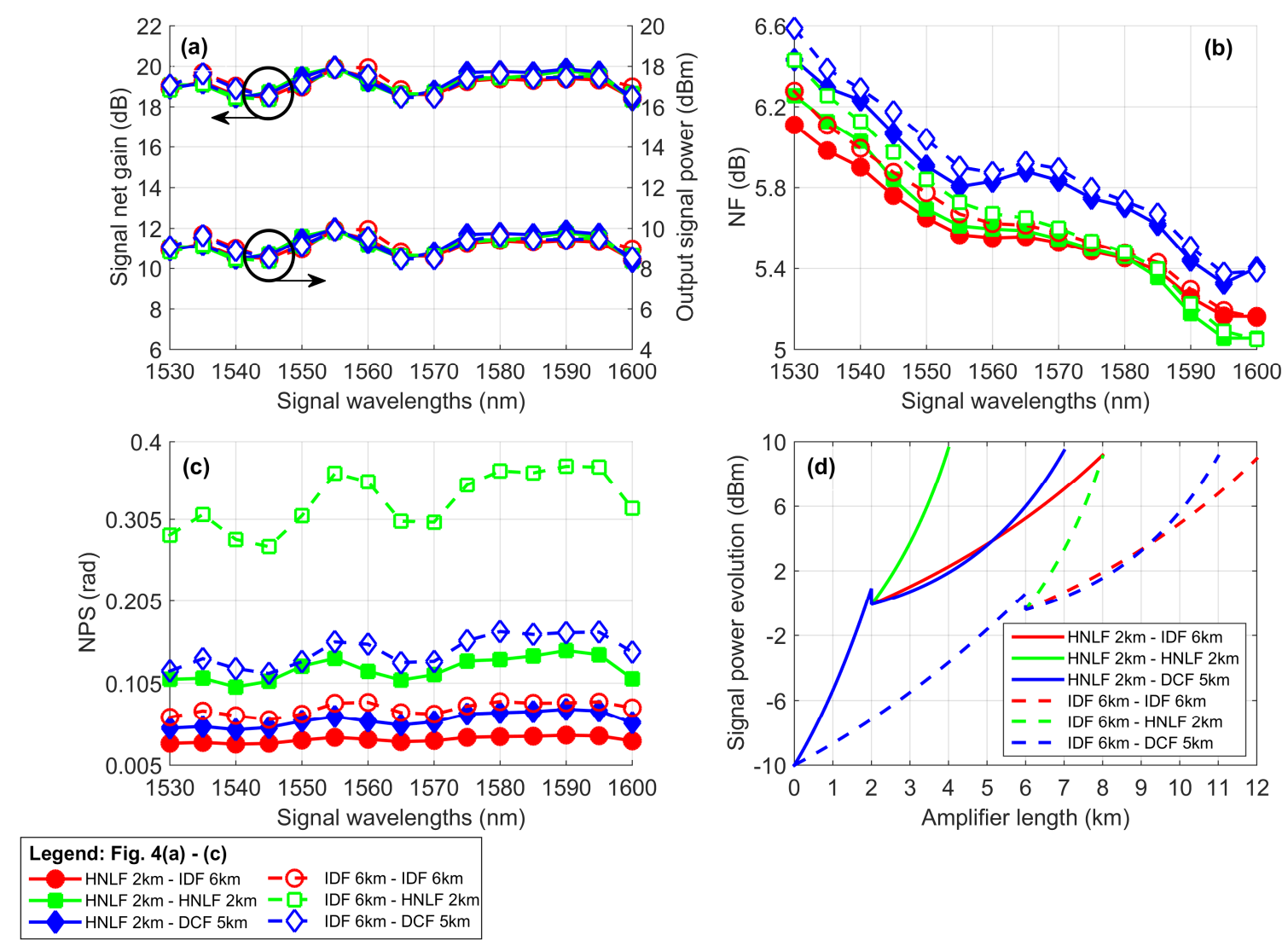

Fig. 4: Performance characterizations of dual stage discrete Raman amplifier (a) Signal net gain and output power (b) noise figure (c) nonlinear phase shift and (d) signal power profile at 1550nm.

\section{CONCLUSIONS}

We have characterized the gain, noise and nonlinear performance of dual stage discrete Raman amplifiers with different combinations of three types of gain fibres: IDF, HNLF and DCF at optimum lengths. It is found that a dual stage discrete Raman amplifier design with $>20 \mathrm{dBm}$ output power and $\sim 6 \mathrm{~dB}$ noise figure is possible with $2 \mathrm{~km} \mathrm{HNLF}$ and $6 \mathrm{~km} \mathrm{IDF}$ as $1^{\text {st }}$ and $2^{\text {nd }}$ stage combination respectively, showing the lowest nonlinear phase shift penalty of all the possible gain fibre combinations under study.

\section{ACKNOWLEDGEMENTS}

This work was funded by EU FP7 ITN programme ICONE (No. 608099).

\section{REFERENCES}

[1] T. Miyamoto et al., "Raman amplification over $100 \mathrm{~nm}$ bandwidth with dispersion and dispersion slope compensation for conventional single mode fiber," in Proc. OFC'02, March 2002, paper TuJ7.

[2] J. Bromage et al., "Raman amplification in the S-band," in Proc. OFC'02, March 2002, paper ThB3.

[3] T. Miyamoto et al., "Highly nonlinear fiber-based lumped fiber Raman amplifier for CWDM transmission systems," J. Lightwave Technol., vol.23, no.11, pp.3475-3483, Nov. 2005.

[4] I. Nasieva et al., "Nonlinearity management in fibre links with distributed amplification," in Electronics Letters, vol. 39, no. 11, pp. 856-857, May 2003.

[5] K. Mukasa et al., "Dispersion compensating fiber used as a transmission fiber: inverse/reverse dispersion fiber," J. Opt. Fiber Commun., vol. 3, no. 5, pp. 292-339, Oct. 2006.

[6] D. Hamoir et al. "Optimized, two-stage architecture for Raman amplifiers," in Proc. OAA'00, July 2000, vol. 44, pp. 61-63, paper OMD8.

[7] S. A. E. Lewis et al., "Characterization of double Rayleigh scatter noise in Raman amplifiers," in IEEE Photonics Technol. Lett., vol. 12, no. 5, pp. 528-530, May 2000.

[8] M. A. Iqbal et al., "Performance improvement of broadband distributed Raman amplifier using bidirectional pumping with first and dual order forward pumps, " in Proc. ICTON 2016, Trento, Italy, July 2016, pp. 1-4. 